\title{
A Covalently Imprinted Photonic Crystal for Glucose Sensing
}

\author{
Fei Xue, Ting-rui Duan, Shu-yue Huang, Qiu-hong Wang, Min Xue, and Zi-hui Meng
}

School of Chemical Engineering and Environment, Beijing Institute of Technology, Beijing 100081, China

Correspondence should be addressed to Zi-hui Meng; m_zihui@yahoo.com

Received 27 April 2013; Revised 9 June 2013; Accepted 9 June 2013

Academic Editor: Amir Kajbafvala

Copyright (C) 2013 Fei Xue et al. This is an open access article distributed under the Creative Commons Attribution License, which permits unrestricted use, distribution, and reproduction in any medium, provided the original work is properly cited.

\begin{abstract}
We demonstrate a glucose-sensing material based on the combination of photonic crystal templating and a molecular-imprinting technique. In the presence of the target molecule glucose, poly $(N$-isopropylacrylamide-co-2-hydroxyethyl methacrylate) hydrogel with pendent phenylboronic acid groups was synthesized in the void of a poly(methyl methacrylate) (PMMA) colloidal crystal. After removing the PMMA photonic crystal template and glucose molecules, a 3D-ordered porous covalently imprinted photonic crystal (CIPC) hydrogel was created. The unique 3D-ordered porous hydrogel revealed optical changes in response to glucose concentration. At $\mathrm{pH}=11$ and $37^{\circ} \mathrm{C}$, the diffraction of CIPC redshifted from $725 \mathrm{~nm}$ to $880 \mathrm{~nm}$ in response to $20 \mathrm{mmol} \mathrm{L}^{-1}$ glucose. Due to the covalently imprinted recognizer, boronic acid group, the selectivity of the CIPC towards glucose over $D$-ribose and $L$-rhamnose was improved significantly.
\end{abstract}

\section{Introduction}

The prevalence of type 1 diabetes continues to increase worldwide. Real-time continuous glucose monitoring (RT-CGM) is crucial for diabetic care, which can lead to better treatment decisions, improve metabolic control, and decrease the incidence and progression of diabetic complications [1-6]. For this reason, many different analytical methods for RT-CGM have been developed, which are mainly electrochemical [79] and optical $[3,10,11]$. Photonic crystals are highly ordered materials with periods on the scale of visible light and exhibit unique structural color on the basis of Bragg diffraction and lattice spacing. It is found that the diffraction wavelength of photonic crystals is dependent on their lattices. Various approaches have been explored for making bioresponsive photonic crystal hydrogels for glucose sensing. Honda et al. developed a totally synthetic colorimetric glucose-sensing system composed of glucose-responsive hydrogel particles in an inverse opal polymer membrane [12]. The volume of the hydrogel particles can change reversibly as the glucose concentration varies and exhibits a reversible change in the color and the peak intensity of the reflection spectra. Asher's group has also made remarkable contributions to glucose-responsive photonic crystals. They incorporated a crystalline colloidal array (CCA) into a polyacrylamide [13] or poly(vinyl alcohol) [14] hydrogel with pendent boronic acid groups, and the polymerized crystalline colloidal array (PCCA) diffraction wavelength was dependent on the hydrogel volume [15]. Bazin and Zhu prepared core-shell microspheres and assembled them into a crystalline colloidal array (CCA) [3]. The core-shell microspheres were functionalized with 3-aminophenylboronic acid to make them responsive to glucose. More recently, Ayyub et al. investigated onedimensional photonic crystals as a sensitive carbohydrate sensor by modifying polystyrene-b-poly(2-vinyl pyridine) films with 2-(bromomethyl)phenylboronic acid [11]. Besides boronic acid, glucose oxidase (GOx) was also attached to the PCCA and glucose caused the PCCA to swell and redshift the diffraction wavelength [16]. Although photonic crystals now attract more and more attention, their selectivity is not promising. Immobilization of enzymes might improve the selectivity, but natural receptors usually deteriorate quickly due to the variation of temperature, $\mathrm{pH}$, humidity, and toxic chemicals.

Molecular imprinting is a state-of-the-art technique to artificially mimic natural and biological receptors $[17,18]$. Molecularly imprinted polymers (MIPs) have several advantages, including stability under harsh physical and chemical conditions, easy preparation, and more importantly the ability to be "tailored" recognition materials for the target 
analytes. MIPs have attracted considerable attention as a recognition element with high selectivity for glucose sensors. Chen et al. reported an MIP that could be used to measure glucose concentrations in complex biological media. At alkaline $\mathrm{pH}$, the metal-complexing polymer "catches" glucose and instantly releases protons in proportion to the glucose concentration over a clinically relevant range ( 0 to $\left.25 \mathrm{mmol} \mathrm{L}^{-1}\right)$ [19]. Malitesta et al. electropolymerized a glucose-imprinted poly $(O$-phenylenediamine) polymer, which was employed as the recognition element of a QCM biomimetic sensor for glucose [20]. Wu's group designed a kind of molecularly imprinted hybrid microgels that can optically monitor glucose levels with high sensitivity and selectivity in complex media at physiological pH [21]. Recently, Li's group coupled molecular imprinting with photonic crystal to create selfreporting specific sensors to detect protein, medicines and some biomarkers [22-25]. However, specificity cannot be guaranteed by traditional noncovalent imprinting protocol, and in most cases, the diffraction shifts are not remarkable. We here report on a covalently imprinted photonic crystal (CIPC) for glucose sensing. The use of boronic acid derivatives to sense diol-containing substances has been exploited for a long time. Boronic acid can bind cis diol-containing substances through covalent bonds. Our CIPC glucose-sensing hydrogel also utilized boronic acid groups as recognition elements. In order to improve the selectivity of photonic crystal sensor, covalently imprinting was introduced into the photonic crystal hydrogel. Boronic acid derivatives have been polymerized in the presence of glucose to create imprinted glucose-binding sites. Polymer hydrogel volume changes could be actuated by boronic acid-glucose complexation. The CIPC volume changes alter the photonic crystal spacing, which alters the Bragg diffraction condition.

\section{Materials and Methods}

2.1. Materials. Acrylamide (AM), acrylic acid (AA), 2,2'azobisisoheptonitrile (ABVN), 2-hydroxyethyl methacrylate (HEMA), $N$-isopropylacrylamide (NIPA), 2,2-diethoxyacetophenone (DEAP), 4-ethylene phenylboronic acid (4EPBA), and $N, N^{\prime}$-methylenebisacrylamide (BIS) were all purchased from Tokyo Chemical Industry (Tokyo, Japan). Methyl methacrylate (MMA), glycine, glucose, D-ribose, $L$-rhamnose, acetonitrile, dimethyl sulfoxide (DMSO) and acetonitrile were all from local suppliers and were used directly.

2.2. Instruments. The diffraction spectra were detected using an Avaspec-2048TEC optical fiber spectrometer (Avantes, Netherlands). UV polymerization was carried out in a SCIENTZ D3-IIUV cross-linker (Ning Bo, China). The assembling of PMMA colloidal crystal was carried out in a Safe HWS-150 incubator (Ning Bo, China). SEM images were taken using a HITACHI S4800 field emission scanning electron microscope (Tokyo, Japan) with an accelerating voltage of $15 \mathrm{kV}$. A Shimadzu LC-20AT HPLC system on a Promosil silica column $(4.6 \times 250 \mathrm{~mm}$, Agela Technologies, Tianjing, China) with an evaporative light-scattering detector

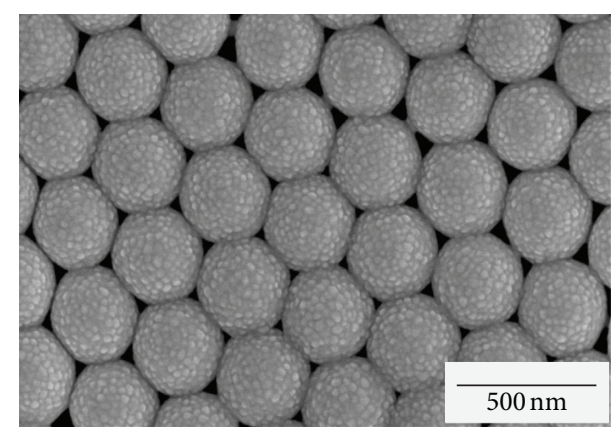

FIGURE 1: SEM image of the PMMA colloidal crystal.

(ELSD) was used to quantitatively characterize the imprinting effect of MIPs. The mobile phase was acetonitrile/water (2/1, $\mathrm{v} / \mathrm{v}$ ) with a flow rate of $1 \mathrm{~mL} \mathrm{~min}^{-1}$, and the injection volume was $20 \mu \mathrm{L}$.

2.3. Preparation of PMMA Colloidal Crystal. Poly(methyl methacrylate) (PMMA) monodispersed colloidal particles $(280 \mathrm{~nm})$ were synthesized using a modification of published methods $[26,27]$. MMA $(30 \mathrm{~mL})$ and deionized water $(250 \mathrm{~mL})$ were mixed in a 4 -neck flask at $80^{\circ} \mathrm{C}$, stirred at $300 \mathrm{r} \mathrm{min}^{-1}$, and bubbled with nitrogen. After equilibrating to $80^{\circ} \mathrm{C}, 15 \mathrm{~mL}$ water containing $0.6 \mathrm{~g}$ potassium persulfate was added to the flask to initiate polymerization. Monodisperse PMMA particles with a diameter of approximately $280 \mathrm{~nm}$ were obtained after 40 minutes (Figure 1). The resulting particles were washed by centrifugation and rinsed at least three times with deionized water.

The PMMA colloids were self-assembled into colloidal crystals via a vertical deposition method as a template of the opal structure. Typically, glass slides were immerged in $\mathrm{H}_{2} \mathrm{SO}_{4} / \mathrm{H}_{2} \mathrm{O}_{2}$ mixture $(7: 3, \mathrm{~V} / \mathrm{V})$ for $12 \mathrm{~h}$, then rinsed with deionized water in ultrasonic bath three times, and dried before use. Mono-disperse PMMA particles were fully dispersed in deionized water $(0.25 \%-0.35 \%$, w/w), which were added to a clean Petri dish for the colloidal crystal formation. Clean glass slides were placed vertically into the Petri dish for colloidal crystal growth. After complete volatilization of the deionized water, a colloidal crystal of PMMA particles was formed on both sides of each glass slide.

2.4. Preparation of CIPC Films. Three MIPs pre-polymerization solutions, each with different recipes (see Table 1) were prepared. Take MIPs recipe (B), for instance, glucose $(0.17 \mathrm{mmol})$, covalent functional monomer 4-APBA (0.17 mmol), backbone monomers HEMA ( $1.5 \mathrm{mmol})$, and NIPA $(1.5 \mathrm{mmol})$ were dissolved into a mixture of $1.5 \mathrm{~mL}$ DMSO. Then, crosslinker BIS $(0.254 \mathrm{mmol})$ and initiator ABVN $(12.68 \mu \mathrm{mol})$ were added and the mixture was fully deoxygenated by nitrogen purging for $10 \mathrm{~min}$. The prepolymerization solution was layered carefully on the PMMA photonic crystal template so that it can infiltrate into the CCA and then polymerize at $60^{\circ} \mathrm{C}$ to form hydrogel film. The film was immersed into acetone to remove the PMMA photonic crystal template, followed by the removal of glucose by acidic 
TABLE 1: Composition of different MIPs prepolymerization solutions.

\begin{tabular}{|c|c|c|c|c|c|c|}
\hline MIPs & $\begin{array}{l}\text { Template } \\
\text { (mmol) }\end{array}$ & $\begin{array}{c}\text { Monomers } \\
(\mathrm{mmol})\end{array}$ & $\begin{array}{l}\text { Crosslinker } \\
(\mathrm{mmol})\end{array}$ & $\begin{array}{c}\text { Initiator } \\
(\mathrm{mmol})\end{array}$ & $\begin{array}{l}\text { Solvent } \\
(\mathrm{mL})\end{array}$ & Polymerization \\
\hline A & Glucose (0.7) & $\begin{array}{c}4 \text {-EPBA }(0.7)+\text { AM } \\
(1.4)+\text { HEMA }(1.4)\end{array}$ & BIS (0.0162) & DEAP $(0.05)$ & Acetonitrile (2) & UV irradiation $4^{\circ} \mathrm{C}$ \\
\hline B & Glucose (0.17) & $\begin{array}{c}\text { 4-EPBA }(0.17)+\text { NIPA } \\
(1.5)+\text { HEMA }(1.5)\end{array}$ & BIS $(0.254)$ & $\mathrm{ABVN}(0.01)$ & DMSO (1) & $60^{\circ} \mathrm{C}$ \\
\hline $\mathrm{C}$ & Glucose (0.17) & $\begin{array}{c}\text { 4-EPBA }(0.17)+ \\
\text { HEMA }(1.5)\end{array}$ & BIS (0.317) & $\operatorname{DEAP}(0.5)$ & Acetonitrile (3) & UV irradiation $4^{\circ} \mathrm{C}$ \\
\hline
\end{tabular}

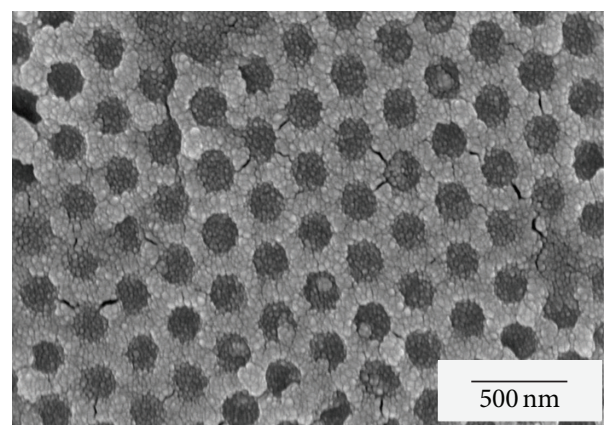

FIGURE 2: SEM image of the macroporous structure of the CIPC film.

solution to give the CIPC hydrogel. A nonimprinted photonic crystal (NIPC) was prepared in the same procedure in the absence of glucose.

The three CIPC films were dried in a vacuum oven over night and then were exposed to glucose to evaluate their adsorption capability and selectivity. Typically, glucose solutions $\left(20 \mathrm{mmol} \mathrm{L}^{-1}\right)$, prepared in $1 \mathrm{~mL}$ methanol/deionized water $(6: 4, \mathrm{v} / \mathrm{v})$, were incubated with $10 \mathrm{mg}$ CIPC film for $1 \mathrm{~h}$ at room temperature. After incubation, the residual concentration of glucose in the solution was determined by HPLC.

\section{Results and Discussion}

Figure 1 shows the SEM images of the used PMMA colloidal crystal, in which the PMMA particle size is about $280 \mathrm{~nm}$. From Figure 1, we can see that the PMMA colloidal crystal has high ordering. After removing the PMMA photonic crystal template, highly ordered macroporous structure could be observed using SEM (Figure 2). More importantly, the ordering of the macroporous structure was kept well. In order to optimize the recipe of covalently imprinted molecules, we tried three different recipes. The adsorption rates of the three kinds of MIPs in $20 \mathrm{mmol} \mathrm{L}^{-1}$ glucose were measured via HPLC. It was revealed in Figure 3 that even though recipe $\operatorname{MIPs}(\mathrm{A})$ and $\operatorname{MIPs}(\mathrm{C})$ had better adsorption rates, recipe MIPs(B) had better imprinting effect among the three MIPs. As a result, we chose recipe MIPs(B) to study the following absorption and sensing properties of the CIPC.

Boronic acid is a classic saccharide receptor [28, 29], which can form reversible covalent bonds with glucose. Herein, 4-EPBA which contains a boronic acid functional

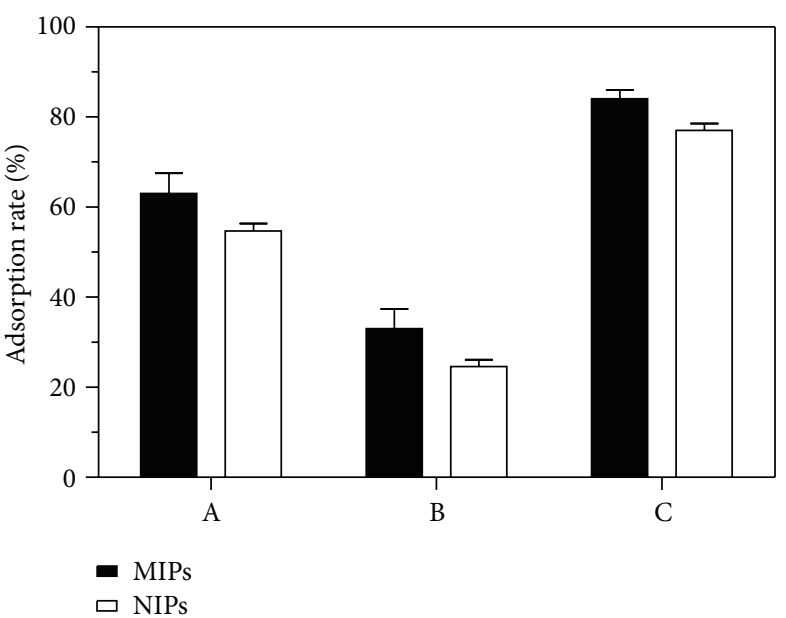

FIGURE 3: Adsorptivity of the MIPs of different recipes.

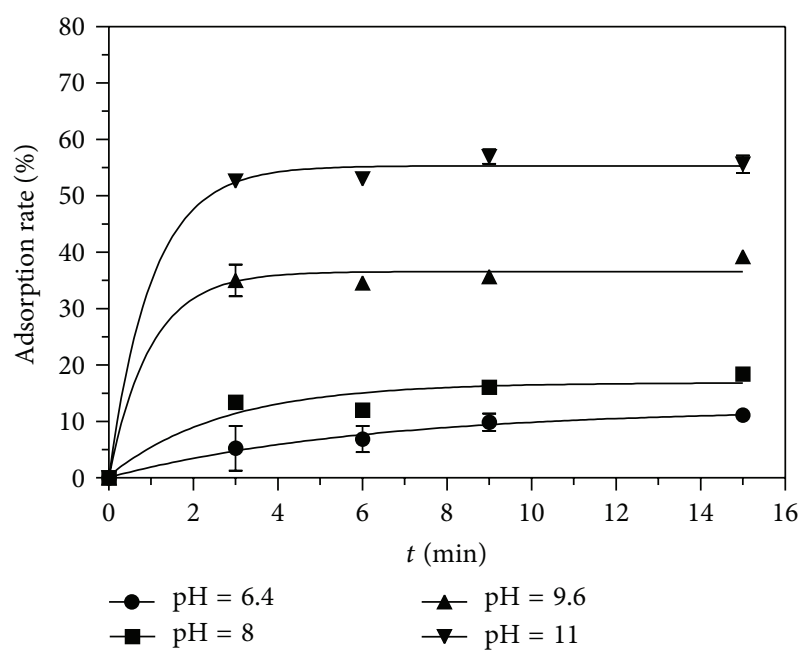

FIgURE 4: Adsorptivity of the CIPC towards $10 \mathrm{mmol} \mathrm{L}^{-1}$ glucose under different $\mathrm{pH}$.

group was used as the covalent recognizer which forms boronate with glucose. The formation of boronate is dependent on $\mathrm{pH}$, ionic strength, and temperature. Thus, a glucose sensor should work in an optimal environment. The adsorptivity of CIPC was measured by exposure to varying concentrations of glucose $\left(0\right.$ to $\left.20 \mathrm{mmol} \mathrm{L}^{-1}\right)$, followed by the detection of glucose in the residual solution using HPLC. 


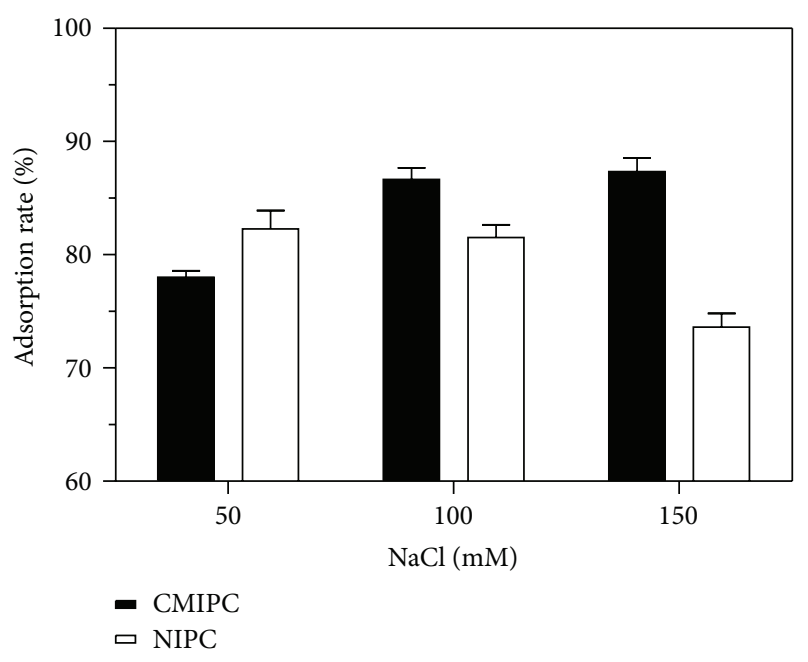

FIgURE 5: Adsorptivity of the CIPC towards $10 \mathrm{mmol} \mathrm{L}^{-1}$ glucose under different ion strength.

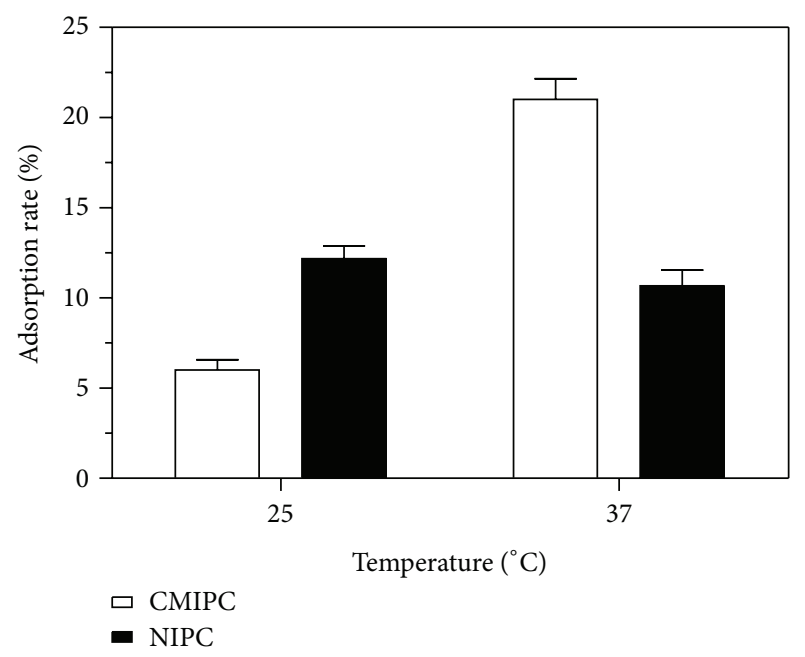

Figure 6: Adsorptivity of the CIPC towards $10 \mathrm{mmol} \mathrm{L}^{-1}$ glucose at room temperature and body temperature.

Figure 4 shows that the adsorption kinetics of glucose on $\mathrm{CIPC}$ in different $\mathrm{pH}$ condition. As shown in Figure 4, within about 3 minutes soaking in a solution of $10 \mathrm{mmol} \mathrm{L}^{-1}$ glucose the adsorption already reaches the adsorption equilibrium. The presence of macropores provides high specific surface area, more interaction sites, and can also offer increased mass transport. In addition, the response of the sensor to glucose decreases as the $\mathrm{pH}$ increases. The adsorptivity of $10 \mathrm{mmol} \mathrm{L}^{-1}$ glucose at $\mathrm{pH} 11$ is 5 times higher than that of at $\mathrm{pH}$ 6.4. An alkaline environment is favorable for boronate formation between glucose and 4-EPBA.

The adsorptivity of glucose to the CIPC also increases with increasing ionic strength (Figure 5). The effects of temperature on glucose sensing were also investigated by measuring the adsorptivity at room temperature and body temperature. The result in Figure 6 revealed that the CIPC had better selectivity at $37^{\circ} \mathrm{C}$ than at $25^{\circ} \mathrm{C}$. The sensitivity is considerably better at body temperature, which is beneficial for the detection of glucose in bodily fluid. In short, the CIPC can characteristically detect glucose under alkaline conditions, at high ionic strength, and human body temperature. With regard to the imprinting effect, even though the NIPC also contains substantial amount of 4-EPBA that enables the NIPC to absorb significant amount of glucose, the imprinting process gave molecular "memory" to the hydrogel matrix. This gives the CIPC not only the 4-EPBA covalent recognizer but also the imprinted pockets that possess molecular "memory" for glucose. Therefore, the CIPC absorbs more glucose than NIPC. For $10 \mathrm{mmol} \mathrm{L}^{-1}$ glucose at $\mathrm{pH} 11$, the absorptivity of the CIPC is more than 3 times higher than that of the NIPC.

The CIPC swelled upon glucose adsorption at physiological ionic strengths. As seen in Figure 7(a), in the absence of glucose, the CIPC diffracts visible light at $725 \mathrm{~nm}$, and the diffraction redshifted gradually upon the addition of glucose. When the glucose concentration reached $20 \mathrm{mmol} \mathrm{L}^{-1}$, which is clinically indicative of serious diabetes, the CIPC diffracts visible light at $880 \mathrm{~nm}$, giving a redshift of $155 \mathrm{~nm}$. In the case of NIPC, a redshift of less than $60 \mathrm{~nm}$ was observed (Figure 7(b)). After adsorption of glucose, a charged complex forms between phenylboronic acid and the 1,2-cis-diol group of glucose, and the lattice of the CIPC hydrogel swells, which results in the diffraction redshift. This swelling of the CIPC is reversible. In addition to adsorptivity, the specificity of the hydrogel towards glucose was also improved significantly by imprinting. Compared with the NIPC which had ratios of redshift of glucose/ribose and glucose/rhamnose of 1.6, the corresponding ratios for CIPC were 3.0 (Figure 8), suggesting that a higher selectivity towards glucose was achieved due to imprinting.

\section{Conclusion}

In summary, a glucose-responsive photonic crystal hydrogel was produced by synthesized hydrogel in the void of PMMA photonic crystal template followed by removing the template. Molecular imprinting improved the selectivity of the photonic crystal-responsive hydrogel towards glucose. The diffraction of the CIPC red-shifted from $725 \mathrm{~nm}$ to $880 \mathrm{~nm}$ in response to $20 \mathrm{mmol} \mathrm{L}^{-1}$ glucose. Improvement of the current CIPC technology is still underway, which aims to optimize the CIPC composition to make the diffraction visible, thus allowing promising clinical application in noninvasive, realtime glucose monitoring.

\section{Conflict of Interests}

None of the authors has ever had direct or indirect financial relations with the commercial identities mentioned in the paper, nor do they declare any other conflict of interests.

\section{Acknowledgments}

This research was supported by NSFC project (20775007). The authors thank Gregory Morgan from Department of 


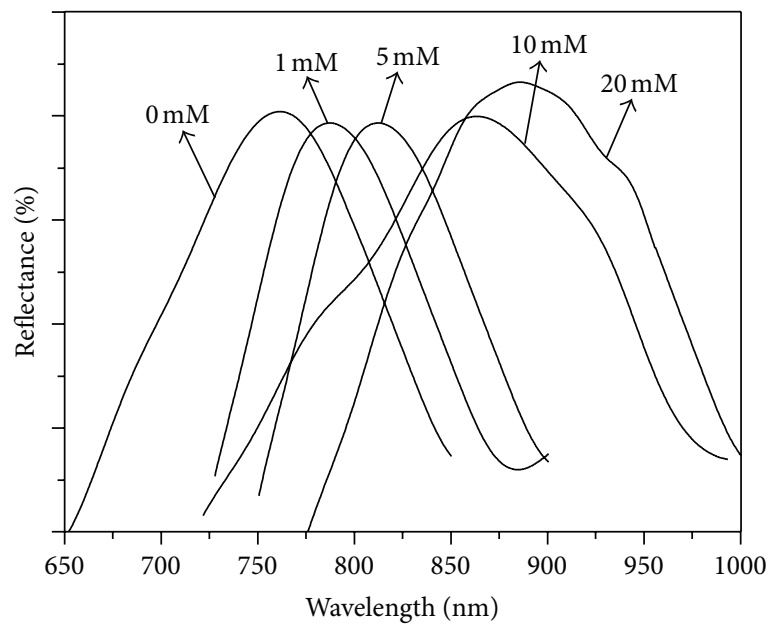

(a)

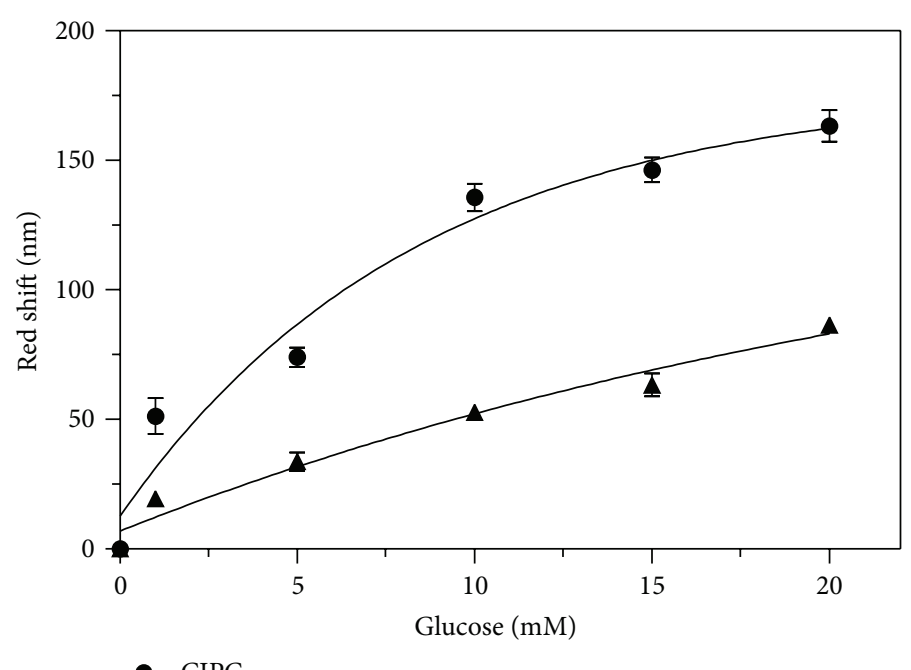

NIPC

(b)

FIGURE 7: Redshift of CIPC (a) and the difference in redshift of CIPC and NIPC (b) in response to glucose. CIPC and NIPC were immersed in glucose solution of different concentration at $\mathrm{pH} 11,150 \mathrm{mmol} \mathrm{L}^{-1}$ ion strength, and $37^{\circ} \mathrm{C}$.

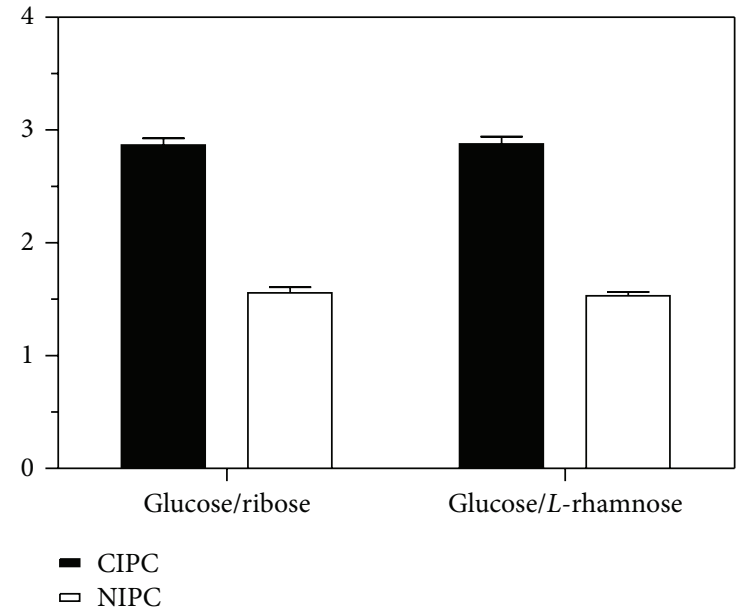

Figure 8: Ratio of the redshift between glucose and other saccharides for the CIPC and the NIPC.

Chemistry, University of Pittsburgh, for his useful comments and language editing which have greatly improved the paper.

\section{References}

[1] S. L. Ellis, R. G. Naik, K. Gemperline, and S. K. Garg, "Use of continuous glucose monitoring in patients with type 1 diabetes," Current Diabetes Reviews, vol. 4, no. 3, pp. 207-217, 2008.

[2] C. M. Girardin, C. Huot, M. Gonthier, and E. Delvin, "Continuous glucose monitoring: a review of biochemical perspectives and clinical use in type 1 diabetes," Clinical Biochemistry, vol. 42, no. 3, pp. 136-142, 2009.

[3] G. Bazin and J. X. Zhu, "Glucose-sensitivity of core-shell microspheres and their crystalline colloidal arrays," Science China Chemistry, vol. 56, no. 1, pp. 65-70, 2013.
[4] A. L. Secher, L. Ringholm, H. U. Andersen, P. Damm, and E. R. Mathiesen, "The effect of real-time continuous glucose monitoring in diabetic pregnancy: a randomized controlled trial," Diabetes Technology \& Therapeutics, vol. 15, pp. A21-A22, 2013.

[5] N. Mauras, L. Fox, K. Englert, and R. W. Beck, "Continuous glucose monitoring in type 1 diabetes," Endocrine, vol. 43, no. 1, pp. 41-50, 2013.

[6] S. Cordua, A. L. Secher, L. Ringholm, P. Damm, and E. R. Mathiesen, "Real-time continuous glucose monitoring during delivery in women with type 1 diabetes," Diabetes Technology \& Therapeutics, vol. 15, pp. A73-A73, 2013.

[7] L. Qu, S. Xia, C. Bian, J. Sun, and J. Han, "A micro-potentiometric hemoglobin immunosensor based on electropolymerized polypyrrole-gold nanoparticles composite," Biosensors \& Bioelectronics, vol. 24, no. 12, pp. 3419-3424, 2009.

[8] P. Scodeller, V. Flexer, R. Szamocki et al., "Wired-enzyme core-shell Au nanoparticle biosensor," Journal of the American Chemical Society, vol. 130, no. 38, pp. 12690-12697, 2008.

[9] N. Quoc Dung, D. Patil, H. Jung, and D. Kim, "A highperformance nonenzymatic glucose sensor made of $\mathrm{CuO}$ SWCNT nanocomposites," Biosensors and Bioelectronics, vol. 42, pp. 280-286, 2013.

[10] B. S. Der and J. D. Dattelbaum, "Construction of a reagentless glucose biosensor using molecular exciton luminescence," Analytical Biochemistry, vol. 375, no. 1, pp. 132-140, 2008.

[11] O. B. Ayyub, M. B. Ibrahim, R. M. Briber, and P. Kofinas, "Self-assembled block copolymer photonic crystal for selective fructose detection," Biosensors and Bioelectronics, vol. 46, pp. 124-129, 2013.

[12] M. Honda, K. Kataoka, T. Seki, and Y. Takeoka, "Confined stimuli-responsive polymer gel in inverse opal polymer membrane for colorimetric glucose sensor," Langmuir, vol. 25, no. 14, pp. 8349-8356, 2009.

[13] S. A. Asher, V. L. Alexeev, A. V. Goponenko et al., "Photonic crystal carbohydrate sensors: low ionic strength sugar sensing," 
Journal of the American Chemical Society, vol. 125, no. 11, pp. 3322-3329, 2003.

[14] Q. Cui, M. M. Ward Muscatello, and S. A. Asher, "Photonic crystal borax competitive binding carbohydrate sensing motif," Analyst, vol. 134, no. 5, pp. 875-880, 2009.

[15] V. L. Alexeev, A. C. Sharma, A. V. Goponenko et al., "High ionic strength glucose-sensing photonic crystal," Analytical Chemistry, vol. 75, no. 10, pp. 2316-2323, 2003.

[16] M. Kamenjicki and S. A. Asher, "Epoxide functionalized polymerized crystalline colloidal arrays," Sensors and Actuators B, vol. 106, no. 1, pp. 373-377, 2005.

[17] G. Wulff, "Molecular imprinting in cross-linked materials with the aid of molecular templates - a way towards artificial antibodies," Angewandte Chemie International Edition in English, vol. 34, no. 17, pp. 1812-1832, 1995.

[18] C. Baggiani, C. Giovannoli, L. Anfossi, C. Passini, P. Baravalle, and G. Giraudi, "A Connection between the binding properties of imprinted and nonimprinted polymers: a change of perspective in molecular imprinting," Journal of the American Chemical Society, vol. 134, no. 3, pp. 1513-1518, 2012.

[19] G. Chen, Z. Guan, C.-T. Chen, L. Fu, V. Sundaresan, and F. H. Arnold, "A glucose-sensing polymer," Nature Biotechnology, vol. 15, no. 4, pp. 354-357, 1997.

[20] C. Malitesta, I. Losito, and P. G. Zambonin, "Molecularly imprinted electrosynthesized polymers: new materials for biomimetic sensors," Analytical Chemistry, vol. 71, no. 7, pp. 1366-1370, 1999.

[21] W. Wu, J. Shen, Y. Li, H. Zhu, P. Banerjee, and S. Zhou, "Specific glucose-to-SPR signal transduction at physiological pH by molecularly imprinted responsive hybrid microgels," Biomaterials, vol. 33, no. 29, pp. 7115-7125, 2012.

[22] W. Zhu, S. Tao, C.-A. Tao et al., "Hierarchically imprinted porous films for rapid and selective detection of explosives," Langmuir, vol. 27, no. 13, pp. 8451-8457, 2011.

[23] D. Xu, W. Zhu, Y. Jiang et al., "Rational design of molecularly imprinted photonic films assisted by chemometrics," Journal of Materials Chemistry, vol. 22, no. 32, pp. 16572-16581, 2012.

[24] X. Hu, G. Li, J. Huang, D. Zhang, and Y. Qiu, "Construction of self-reporting specific chemical sensors with high sensitivity," Advanced Materials, vol. 19, no. 24, pp. 4327-4332, 2007.

[25] X. Hu, G. Li, M. Li et al., "Ultrasensitive specific stimulant assay based on molecularly imprinted photonic hydrogels," Advanced Functional Materials, vol. 18, no. 4, pp. 575-583, 2008.

[26] J. C. Lytle, H. Yan, N. S. Ergang, W. H. Smyrl, and A. Stein, "Structural and electrochemical properties of threedimensionally ordered macroporous tin(IV) oxide films," Journal of Materials Chemistry, vol. 14, no. 10, pp. 1616-1622, 2004.

[27] T. Tanrisever, O. Okay, and I. Ç. Sönmezoğlu, "Kinetics of emulsifier-free emulsion polymerization of methyl methacrylate," Journal of Applied Polymer Science, vol. 61, no. 3, pp. 485493, 1996.

[28] Y.-J. Lee, S. A. Pruzinsky, and P. V. Braun, "Glucose-sensitive inverse opal hydrogels: analysis of optical diffraction response," Langmuir, vol. 20, no. 8, pp. 3096-3106, 2004.

[29] C. Ancla, V. Lapeyre, I. Gosse, B. Catargi, and V. Ravaine, "Designed glucose-responsive microgels with selective shrinking behavior," Langmuir, vol. 27, no. 20, pp. 12693-12701, 2011. 

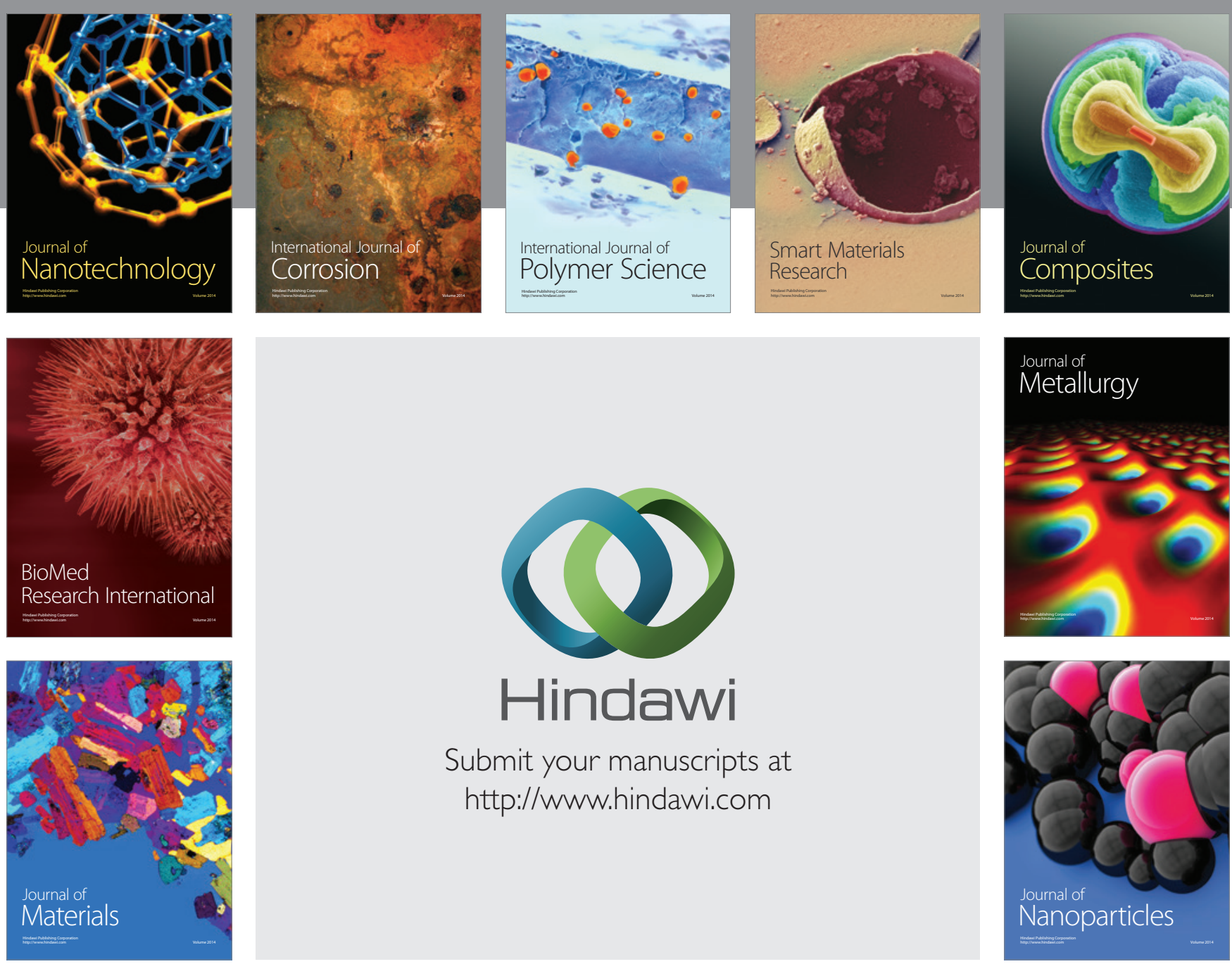

Submit your manuscripts at http://www.hindawi.com
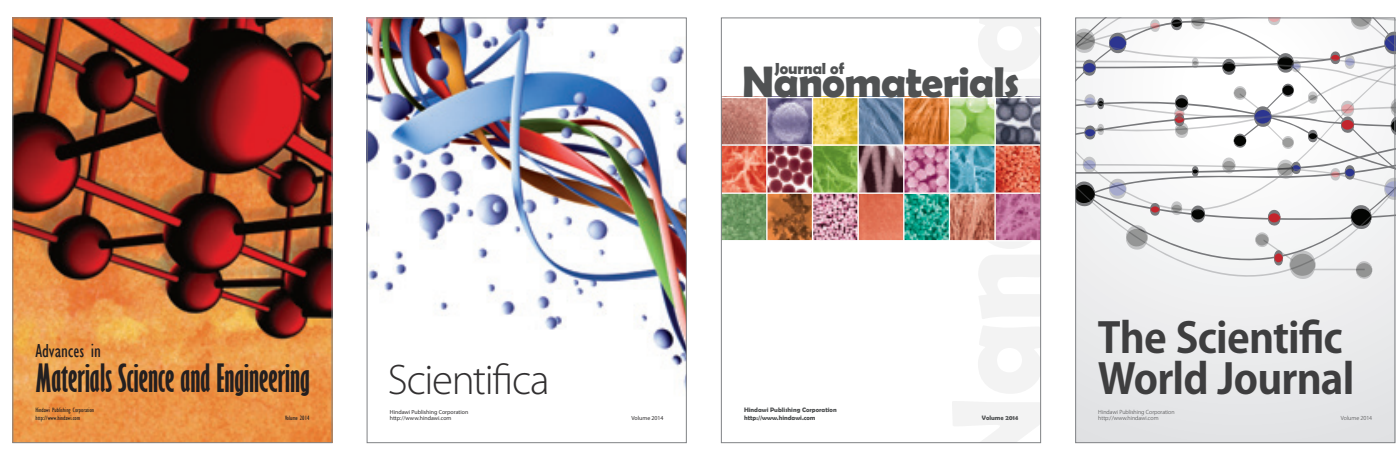

\section{The Scientific World Journal}
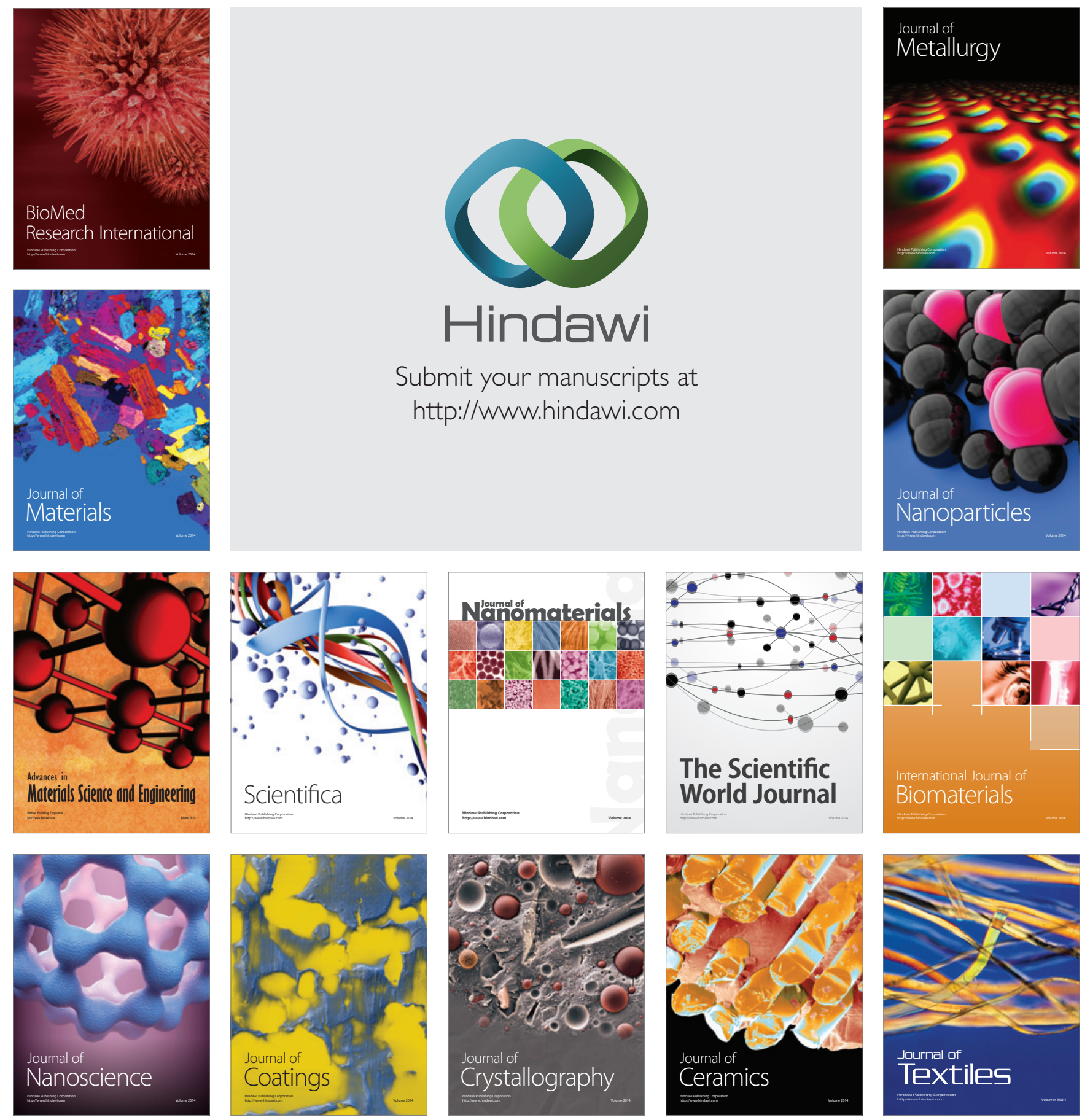